\title{
Qualifying parabolic mirrors with deflectometry
}

J. Burke

W. Li

\section{A. Heimsath \\ C. von Kopylow \\ R. B. Bergmann}

\author{
Bremer Institut für Angewandte Strahltechnik GmbH (BIAS), Klagenfurter Str. 2, 28359 Bremen, Ger- \\ many \\ Vereinigte Elektronik-Werkstätten GmbH (VEW), Edisonstr. 19, 28357 Bremen, Germany \\ Fraunhofer-Institut für Solare Energiesysteme (ISE), Heidenhofstr. 2, 79110 Freiburg, Germany \\ Bremer Institut für Angewandte Strahltechnik GmbH (BIAS), Klagenfurter Str. 2, 28359 Bremen, Ger- \\ many \\ Bremer Institut für Angewandte Strahltechnik GmbH (BIAS), Klagenfurter Str. 2, 28359 Bremen, Ger- \\ many
}

Phase-measuring deflectometry is a full-field gradient technique that lends itself very well to testing reflective optical surfaces. In the past, the industry's interest has been focussed mainly on the detection of defects and ripples, since it is easy to achieve sensitivity in the nm range. On the other hand, attempts to reconstruct the absolute surface shape from the gradient map have been plagued by systematic errors that accumulate to unacceptable uncertainties during data integration. Recently, thanks to improved measurement and evaluation techniques, the state of the art in absolute surface measurement has reached a level of maturity that allows its practical usage in precision optical manufacturing and qualification systems. We demonstrate the techniques, and the progress, by way of results from mirrors for telescopes, solar concentrators, and precision laboratory assemblies.

[DOI: http://dx.doi.org/10.2971/jeos.2013.13014]

Keywords: Deflectometry, gradient integration, optics testing, absolute surface metrology

\section{INTRODUCTION}

Deflectometry utilises the deformation and displacement of a sample pattern after reflection from a test surface to infer the surface slopes [1,2]. We use sinusoidal fringe patterns with phase stepping [3] and hierarchical unwrapping [4], which leads to very low uncertainty for the fringe displacement measurement. The principle is sketched in Figure 1. Integration of the displacement data [5]-[8] allows reconstruction of the absolute surface shape, but the procedure relies on the elimination (or at least complete inclusion) of systematic errors,

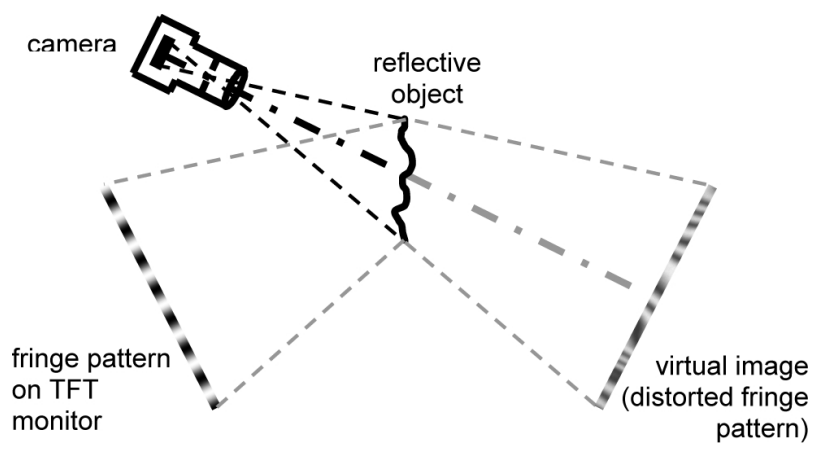

FIG. 1 Measurement principle of phase-measuring deflectometry. A regular fringe pattern is displayed on a monitor; the tested object reflects it toward the camera. Any irregularities in the object give rise to a distortion of the observed fringes, which can be evaluated quantitatively by virtue of the phase-shifting technique. as well as exact information on the measurement geometry and the imaging system. Particular difficulty is encountered in the shape acquisition of parabolic mirrors $[9,10]$, since a linear systematic error takes a parabolic form upon integration and is therefore very hard to separate from the actual surface shape.

This paper is organised as follows: in Section 2 we give a brief explanation for the most common origin of the parabolic shape error; in Section 3 we present several techniques to improve the evaluation and demonstrate them with practical measurement results; Section 4 summarises our view of what the state of the art currently is.

\section{PARABOLIC INTEGRATION ERRORS}

The most prominent systematic integration error arises from insufficient knowledge of the distance between the camera and the object surface. As we will see, it turns out that the integration responds very sensitively to uncertainties in the absolute distance estimate, even when the relative uncertainty is quite small. A measurement model of a deflectometry set-up is shown in Figure 2. It is simplified in that a planar or lowcurvature surface has been assumed and the $y$ co-coordinate has been omitted. These simplifications serve to demonstrate 


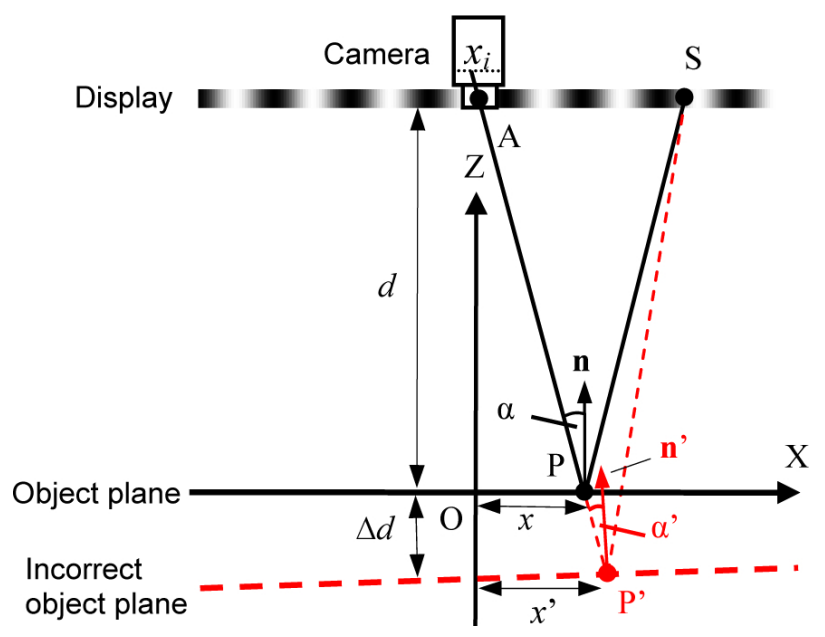

FIG. 2 Simplified 2-D schematic of a measurement set-up. For the definition of variables, see text above.

how the parabolic error comes about; a general measurement is of course in 3-D, and not restricted to quasi-planar surfaces, but this would make the following analysis unnecessarily complicated.

The camera's aperture centre A is located in the centre of the reference pattern display, and the camera's optical axis is normal to the display's surface. The reference coordinate system is $\mathrm{XOZ}, \mathrm{Z}$ being parallel with the camera axis, and $\mathrm{O}$ is the reference point from which the integration starts and whose coordinates must be known. The example object is a plane mirror and aligned parallel to the $\mathrm{X}$ axis. The vision ray of camera pixel $x_{i}$ intersects the specimen's surface at $\mathrm{P}$, and $x$ is the lateral distance from $\mathrm{P}$ orthogonal to the camera's optical axis. The object's distance from the camera is $d$. The "prime" quantities in the plane at $d+\Delta d$ are similar but with an error from incorrect distance measurement or estimation. Correct calculation of the object shape is only possible if $d$ is known exactly so that we obtain the correct surface normal and slope for the starting point of the integration; all other surface points and normals are then obtained by inference. If the distance estimate bears an error $\Delta d$ but the integration is carried out with $d$ as the distance setting, all surface normals - and consequently, the calculated surface shape - will be incorrect.

Tracing back a ray from pixel $x_{i}$, it is reflected in $\mathrm{P}$ and intersects the reference pattern display at $S$, which is uniquely defined by an absolute phase-measurement sequence [4]. These datums allow calculation of the specimen's surface normal vector $\mathbf{n}$ at point $\mathrm{P}$ (or $\mathbf{n}^{\prime}$ at $\mathrm{P}^{\prime}$ ). The surface reflection angle on the surface, $\alpha$, is the bisector between SP and PA (or $\mathrm{SP}^{\prime}$ and $\left.\mathrm{P}^{\prime} \mathrm{A}\right)$, and to calculate the surface slopes we use

$$
\tan \alpha=\frac{x}{d}, \quad \tan \alpha^{\prime} \approx \frac{x}{d+\Delta d^{\prime}}
$$

where for $\alpha^{\prime}$ the assumption is made that $\alpha$ and $\Delta d$ are so small that $x^{\prime}-x$ (the lateral displacement between $\mathrm{P}$ and $\mathrm{P}^{\prime}$ ) is negligible. The error of the calculated surface gradient is then

$$
\Delta g(x)=\tan \alpha^{\prime}-\tan \alpha
$$

and we can insert Eq. (1):

$$
\Delta g(x) \approx \frac{x}{d+\Delta d}-\frac{x}{d}
$$

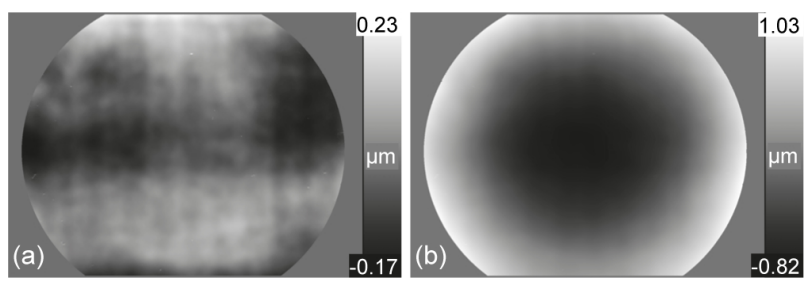

FIG. 3 Height map from measurement of a $100 \mathrm{~mm}$ diameter optical flat $(\lambda / 10$ accuracy). (a) Height distribution calculated with correct object distance $d=400 \mathrm{~mm}$. (b) Height distribution calculated with object distance $(d+\Delta d), \Delta d=0.25 \mathrm{~mm}$.

The absolute surface shape will be obtained from integration of the gradient [5], and the erroneous height distribution $Z^{\prime}(x)$ is expressed as

$$
Z^{\prime}(x)=\int_{0}^{x}\left[g\left(x^{\prime}\right)+\Delta g\right] \cdot d x^{\prime}=Z(x)+\Delta Z(x) .
$$

Because $d \gg \Delta d$, the height error $\Delta Z(x)$ is

$$
\begin{aligned}
\Delta Z(x) & =\int_{0}^{x} \Delta g\left(x^{\prime}\right) \cdot d x^{\prime} \approx \frac{1}{2}\left(\frac{1}{d+\Delta d}-\frac{1}{d}\right) \cdot x^{2} \\
& \approx \frac{\Delta d \cdot x^{2}}{2 d^{2}} .
\end{aligned}
$$

As can now be seen in Eq. (5), the measurement error resulting from incorrect positioning of the specimen has an approximately parabolic shape. It will diminish if $d$ can be increased, but this is seldom possible, as the imaging system must always capture all reflected rays. In most cases, this is a significant constraint on $d$.

Moreover, several approximations have been used in the derivation. The most important simplification is implicit in Eq. (1) and the measurement and object geometry: $\Delta g(x)$ is not an exactly linear function of $x$. As a result, $\Delta Z(x)$ will be predominantly, but not purely parabolic.

To verify the analysis, an experiment has been conducted by measuring a 100-mm diameter optical flat mirror with $\lambda / 10$ surface accuracy. In the experimental set-up, we have $d=400 \mathrm{~mm}$. Using the correct distance $d$ as the input parameter results in the integrated height map shown in Figure 3(a). It is worth emphasising here that the measurement is tolerant of a small tilt of the object (several degrees), as long as the reference pixel still has a distance of $d$. Since the system is typically calibrated with several tilted positions of the same mirror, measurements of tilted surfaces are by definition allowable.

When evaluating the same object with $d+\Delta d=400.25 \mathrm{~mm}$ as the input parameter, the integrated height map shown in Figure 3(b) is obtained.

Whilst the correct map also has some residual measurement errors, as well as genuine flatness error, both of them are almost completely overwhelmed by the large integration error. The measurement error has a clear parabolic structure with a peak-to-valley dynamic range of about $1.7 \mu \mathrm{m}$. Inserting the dimensions of the experiment into Eq. (5) yields an estimate of $1.95 \mu \mathrm{m}$, which shows that the derivation, simple as it is, works in a useful way in practice. 

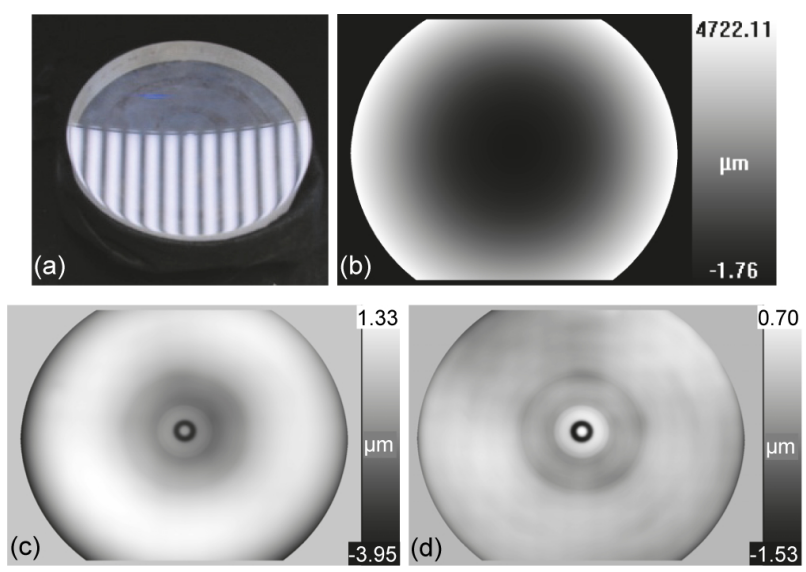

FIG. 4 Measurement of a parabolic telescope mirror: (a) photograph of the mirror with reflected reference pattern; (b) height distribution of the specimen from deflectometric measurement; (c) remaining error after removal of a best-fit sphere with a ROC of $1037.83 \mathrm{~mm}$; (d) remaining error after removal of a best-fit on-axis paraboloid with focal length $517.8 \mathrm{~mm}$.

\section{IMPROVING THE EVALUATION}

It is evident from the previous considerations that $d$ must be estimated and/or measured as accurately as possible, and there are several useful strategies to do this. We will present three of them here in order of increasing accuracy.

\subsection{Rough distance measurement: crossed laser pointers}

This approach uses two laser pointers that are attached to the system near the monitor, so that they can be pointed at the surface under test at an angle. They can be adjusted so that there is one point in space where they cross. This point is then used in the system calibration - which is beyond the scope of this article - and then constitutes a reference and starting datum for the data integration.

\subsubsection{Telescope mirror}

With this system, we measured a circular on-axis parabolic mirror, shown in Figure 4(a). The mirror's lateral diameter is $\mathrm{D}=200 \mathrm{~mm}$, its design radius of curvature (ROC) is 1000 $\mathrm{mm}$. The resulting 3-D data from the deflectometric measurement, shown in Figure 4(b), were compared with a tactile measurement on a coordinate measurement machine (CMM). The ROC from the tactile measurement is $(1038.1 \pm 0.4) \mathrm{mm}$; this is in very good agreement with the reading of $1037.83 \mathrm{~mm}$ that we obtain from the deflectometric measurement. The residuals from the fit to the deflectometric data are displayed in Figure 4(c).

Computing the height error from D and ROC, the results agree to within $1.26 \mu \mathrm{m}$ at the edge of the mirror, where the deflectometric measurement error has its maximum value. The error drops to less than $0.5 \mu \mathrm{m}$ (ROC error $\pm 0.1 \mathrm{~mm}$ ) when the polishing flaw in the centre is not considered in the sphere fit (see Section 3.2). This error is in the range of the CMM's uncertainty, and therefore quite useful in practical optics qualification.

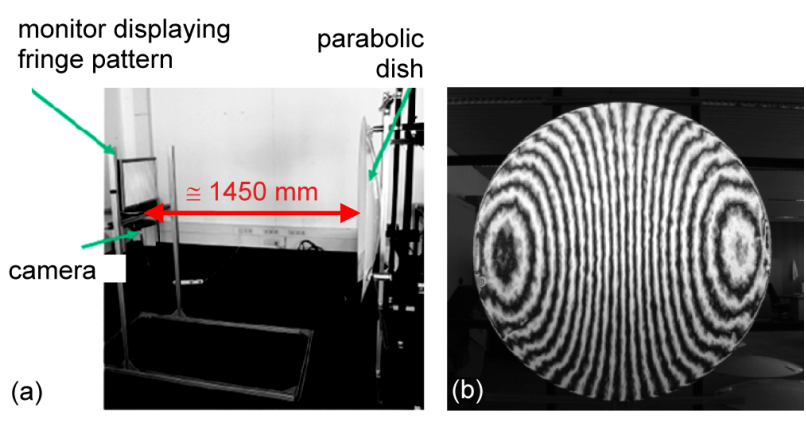

FIG. 5 (a) set-up for solar concentrator measurement; the measurement distance is about twice the mirror's focal length and camera and display are on opposite sides of the mirror's optical axis. (b) fringe image. Irregularities of the surface are visible as irregularities in the fringe pattern; for an impression of the technique's sensitivity, compare this photo with Figure 6(c).

Fitting a paraboloid to the deflectometric height data gives a best-fit paraboloid with a focal length of $517.8 \mathrm{~mm}$. In the residuals map shown in Figure 4(d), traces from the manufacturing process and imperfections in the parabolic profile can clearly be seen. No parabolic error has been subtracted here. As outlined above, this is difficult to do, but would still be possible because the integration error is symmetrical about the reference pixel, whereas the actual error is symmetrical about the optical axis of the tested part. This is where one great advantage of deflectometry comes to bear: unlike interferometric null tests, some freedom exists for positioning and tilting the optic, so that shape errors and systematic errors can be kept separate. Based on the sphere-fit results, the focal length error is about $0.135 \mathrm{~mm}$.

\subsubsection{Solar concentrator}

A solar mirror for concentrating photovoltaic applications with a diameter of $1170 \mathrm{~mm}$ and a design focal length of $725 \mathrm{~mm}$ was measured at the Fraunhofer Institute for Solar Energy [11]. The set-up and a fringe image are shown in Figure 5. The figure is a good illustration of the fact that measurements of collecting optics are not limited by the size of the reference fringe display. In contrast to Figure 1, where the plane surface being measured cannot be larger than half the screen size, no such constraints exist in measuring parabolic dishes. The fringe display and the camera can always be located on either side of the optical axis near the centre of the best-fit ROC (which is approximately twice the focal length), so that concentrators of any size can be measured. To our knowledge, the largest mirror thus far measured with deflectometry is an 8.4-m primary mirror for the Giant Magellan Telescope [12].

Energy output and efficiency of concentrating systems depend strongly on the quality of the optics. Hence, the mirror has been analysed with deflectometry in order to obtain its local surface slope deviations (influencing the reflection of direct solar irradiation and the resulting flux density distribution on the receiving high efficiency III-V PV modules) and its three-dimensional shape, which can be exported into ray tracing software to simulate the element's efficiency. The relevant measurement data are shown in Figure 6.

When measuring a parabolic shape, the expected slope is a 

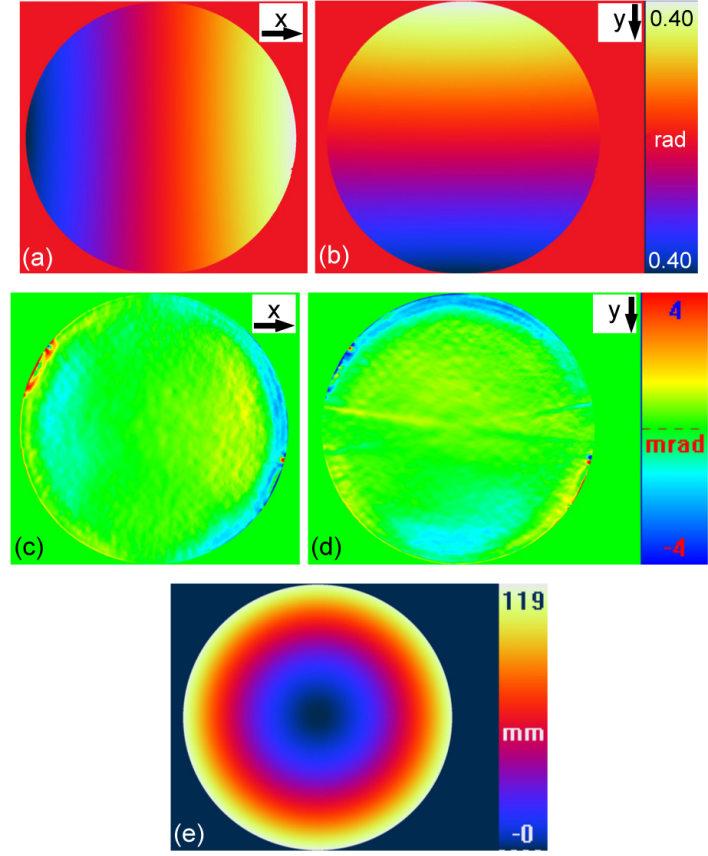

FIG. 6 Deflectometry measurement of a solar concentrator mirror. (a) and (b) surface slopes in $\mathrm{x}$ and $\mathrm{y}$ direction; (c) and (d) slope deviations after subtracting the respective linear ramps. The corresponding calculated standard deviations are $\sigma_{x}=0.8 \mathrm{mrad}$ and $\sigma_{y}=0.9$ mrad. (e) calculated parabolic shape.

linear function; of both co-ordinates in the case of a dish, and of only one in the case of a linear focusing parabolic trough or linear Fresnel collector (which has also been done successfully at FHG-ISE $[13,14])$. Therefore, the range of slopes in the result will be too large to discern small deviations from the design. The errors appear much more prominent, and can be calculated easily, when the slope ramps are subtracted. The data integration issue does not exist here, since deflectometric data are slope data and can be used directly. The relative error of this measurement is $\Delta d / d$, and it is easy to see that even with modest experimental means the error will be smaller than $1 \%$. A common quality parameter for solar mirrors is the rms slope deviation $\sigma$; a good solar mirror should show $\sigma<2 \mathrm{mrad}$. In practice, this means that for a $10-\mathrm{m}$ focal length, most of the power in the concentrator spot will be confined to the central $20 \mathrm{~mm}$.

\subsection{Fine distance estimate: using global shape}

Another possibility for the measurement of known surface shapes is to improve the estimate of $d$ by carrying out a plausibility check of the inferred surface normals [10]. Figure 7 illustrates the principle.

For each surface, the global shape is known to some approximation, and can thus be used as extra information to improve the estimate of $d$. Whenever the assumed $d$ is different from the actual value, the reconstructed surface will acquire an error; and we have seen in Section 2 how sensitive this technique can be. In practice, we set up a cost function whose minimisation guides the improvement of the estimated $d$. The FringeProcessor software developed by BIAS and VEW [15] includes an "IniShape" function that can currently handle pla-
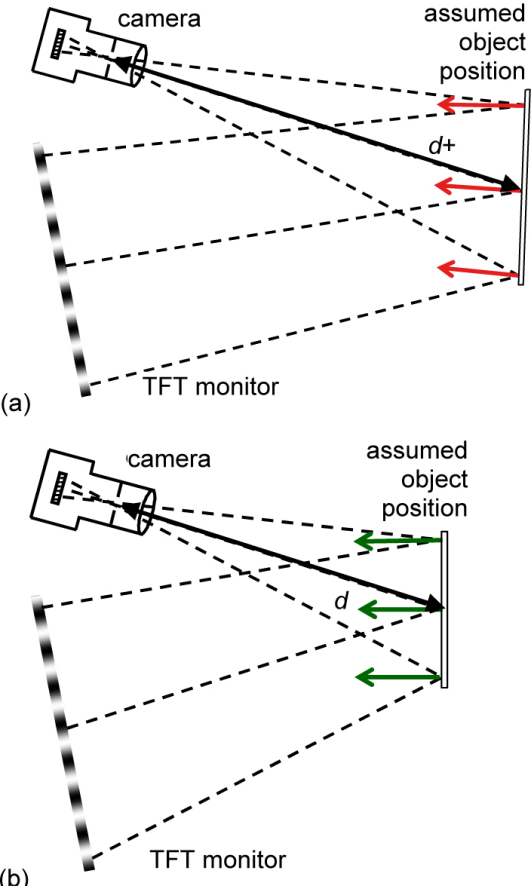

FIG. 7 Improving the estimate for $d$ by matching the surface normals to the expected shape - in this example, a plane. (a) $d+$ is set too large; the surface normals (drawn in red) that are consistent with the measurement geometry are not mutually parallel and the matching surface is curved; (b) the surface normals (drawn in green) are mutually parallel and constitute a best fit for a plane, and the correct $d$ is found.

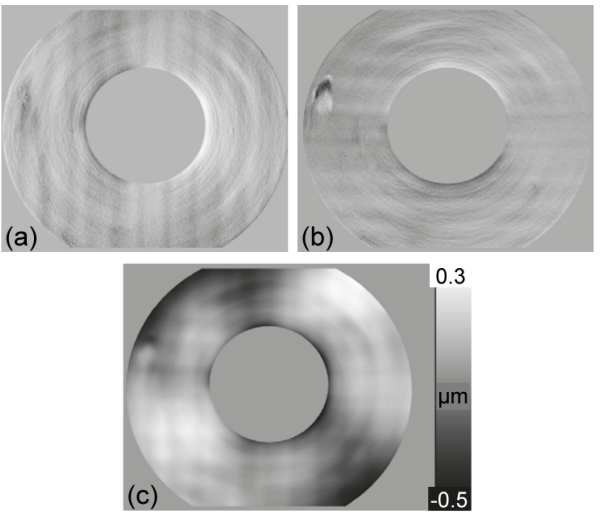

FIG. 8 Results after improving the estimate for $d$ by consistency check of surface normals, using the data from Section 3.1.1 again. (a) and (b) cost function residuals (arbitrary units but same scale in both images) for $x$ and $y$ direction, respectively. (c) total shape deviation from perfect paraboloid after evaluation with improved $d$; compare with Figure $4(d)$

nar, spherical and parabolic surfaces. The shape and slopes of the object are iteratively improved by using all of the calibrated system parameters.

To demonstrate the principle, we re-visit the $200-\mathrm{mm}$ parabolic mirror from Section 3.1.1, but this time we cut out the central region containing the polishing flaws, since it is obvious that the surface normals deviate from the ideal linear distribution there, regardless of the uncertainty of $d$. The residual errors from the optimisation run and the subsequent shape calculation are shown in Figure 8.

It should be stressed that the data integration runs unconstrained once a proper starting datum has been set, so that no 


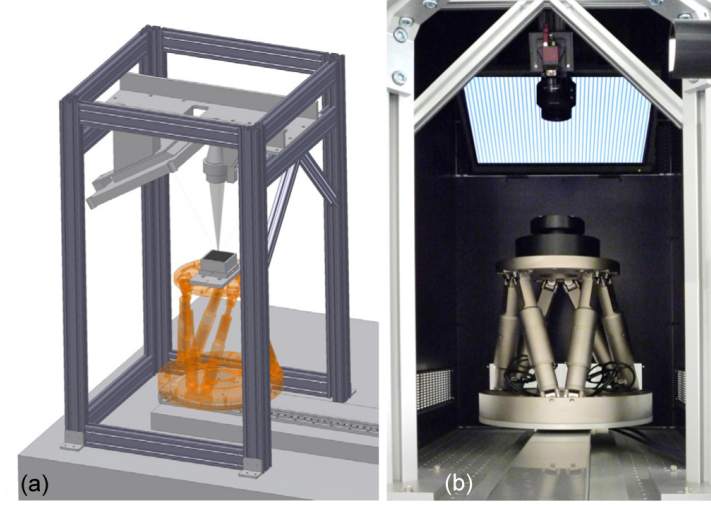

FIG. 9 Low-uncertainty deflectometry system. (a) design sketch showing the light cone of the confocal distance sensor; (b) practical implementation. Note the high-density fringe pattern ensuring high slope sensitivity.

experimental data (other than those we have cut out to begin with) are disregarded.

The focal length of this portion of the mirror comes out as $517.6 \mathrm{~mm}$; this shows that the estimate of $517.8 \mathrm{~mm}$ given above is already quite good, but also that the removal of the central section corresponds to evaluating a slightly different optic.The attractive advantage of the normals consistency check is that it greatly assists the measurement of large parts that do not fit in a pre-calibrated set-up with crossed laser pointers. The possibility to determine $d$ after the measurement can save a fair amount of experimental expense, especially when $d$ is very large. From a practitioner's point of view, the measurement can be taken as soon as the aperture of the optic is covered with reflected fringes.

\subsection{Fine distance estimate: using confocal sensor}

The last technique we want to demonstrate is the use of a confocal white-light sensor, whose distance-measuring uncertainty is known and certified to be a few $\mu \mathrm{m}$. The set-up is calibrated with multiple tilts of a flat mirror whose reference point is kept at exactly the same point in space, at a distance $d_{0}$. This same point must then be in the surface of any object tested to ensure low uncertainty. The set-up is shown in Figure 9.

A measurement of a diamond-turned off-axis parabolic (OAP) mirror was carried out to validate the method. The calculation was then performed using the set distance $d_{0}$; the mirror and the resulting 3-D surface shape are shown in Figure 10. The best-fit paraboloid to this point cloud has a parent focal length of $25.5 \mathrm{~mm}$, which agrees well with the manufacturer tolerances of $(25.4 \pm 0.254) \mathrm{mm}$ of the mirror given by the manufacturer (but since the OAP has a nominal deflection angle of $90^{\circ}$, the effective focal length of this element is $50.8 \mathrm{~mm}$ ). Subtracting the best-fit paraboloid from the 3-D data yields the deviation of the mirror from its nominal shape. This map is shown in Figure 10(c). Another advantage of deflectometry comes to bear here: its very high dynamic range. Whilst the height range of the surface considered here amounts to al-
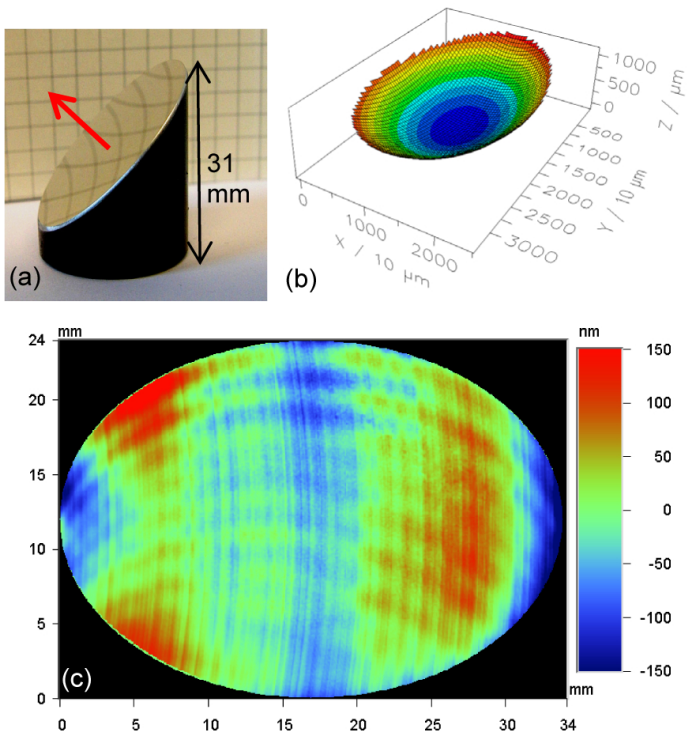

FIG. 10 (a) $90^{\circ}$ off-axis parabolic mirror; alignment with respect to axis of optical distance sensor for deflectometric measurement is indicated by red arrow. (b) measured height data [16]. (c) residual error after subtraction of best-fit paraboloid.
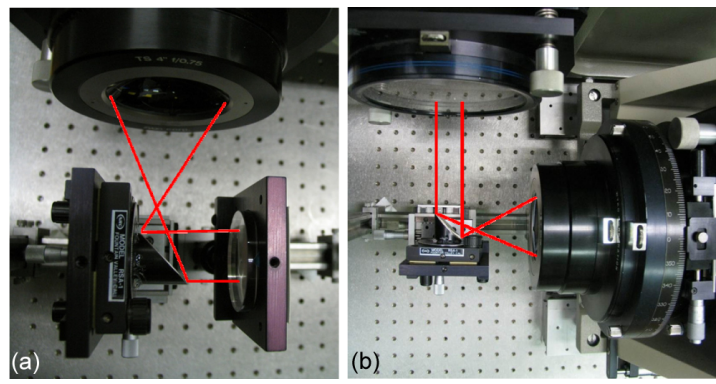

FIG. 11 Interferometric null tests of an OAP. (a) with spherical reference wave and flat return mirror; test wave emanates from focus of OAP; (b) with plane reference wave and spherical return mirror; test wave parallel to optical axis of parent paraboloid.

most $1 \mathrm{~mm}$, it is evident that surface imperfections of several nm can easily be resolved.

A surface of this quality is accessible to interferometric null tests which, despite being much more difficult to set up and carry out than deflectometric measurements, are still the reference standard in optical precision metrology. Such measurements of our test mirror have indeed been carried out at CSIRO's Australian Centre for Precision Optics in Sydney, Australia [17, 18], in two different configurations that are sketched in Figure 11.

Figure 12 presents the deflectometric measurements (appearing circular due to the $\pm 45^{\circ}$ viewing angles, cf. Figure 11).

As can be seen, the agreement between the three measurements is excellent, save for the right edge of the deflectometric result that shows a deviation of slightly over $100 \mathrm{~nm}$. This is not a calibration issue, as the aperture of the mirror is well within that of the calibration mirror used. The error is also independent of the rotation of the mirror in the measurement field. Although the integration instability occurring here could be related to edge artefacts, it is not yet understood and under further investigation. On the whole however, these measurements show that a carefully calibrated and aligned 


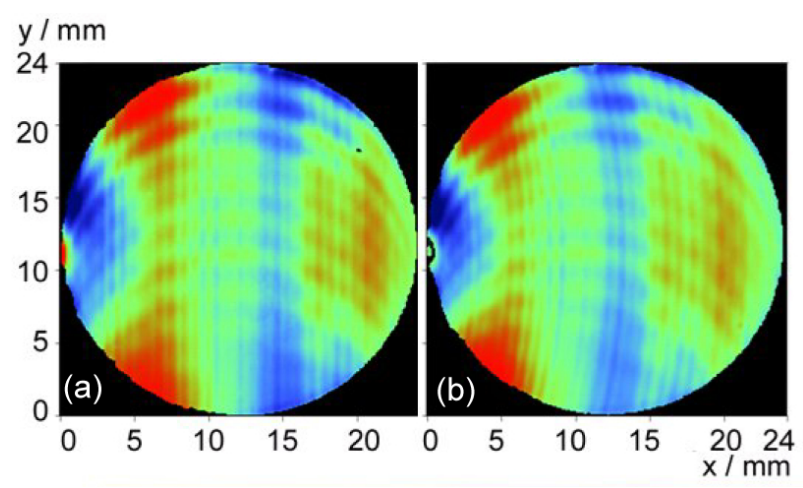

FIG. 12 Error maps from measurements of $90^{\circ}$ off-axis parabolic mirror. (a): interferometric null test with spherical reference wave [17] - cutting traces appear straight; (b): interferometric null test with planar reference wave [18] - cutting traces appear curved.

deflectometric measurement can reach interferometric accuracy.

\section{SUMMARY}

Phase-measuring deflectometry is a surprisingly simple technique for white-light fringe analysis that is evolving from a defect-testing technique towards being useful in industrial metrology applications, including those as yet restricted to the domain of interferometry.

We have presented some examples of parabolic-mirror measurements that demonstrate the increasing impact of deflectometry in optics measurement. In order to tackle the general problems of unknown object distance and ensuing data integration errors, we have proposed three techniques for measuring or estimating the distance as accurately as possible. Each of them uses a different piece of additional information, and the achievable uncertainty has been seen to depend on the experimental and/or computational expense. Since the highest accuracy is not always required, it is a viable strategy to select the simplest approach that will comply with the specifications.

Careful calibration and set-up of deflectometric measurement systems is equally as important as with interferometers, but much easier and quicker to do. Deflectometry is a full-field technique that does not require lengthy alignment, is quite insensitive to environmental disturbances, and almost arbitrarily scalable in the case of light-collecting optics.

\section{ACKN OWLEDGEMENTS}

We thank the BMU (German Federal Ministry for the Environment, Nature Conservation and Nuclear Safety), funding the KomGen project, and Norbert Köpp (VEW GmbH), for support of parts of this work.

\section{References}

[1] C. Faber, E. Olesch, R. Krobot, and G. Häusler, "Deflectometry challenges interferometry: the competition gets tougher!," Proc. SPIE 8493, 84930R (2012).

[2] W. Li, M. Sandner, A. Gesierich, and J. Burke, "Absolute optical surface measurement with deflectometry," Proc. SPIE 8494, 84940G (2012).

[3] J. Burke, "Phase Decoding and Reconstruction," in Optical Methods for Solid Mechanics E. Hack, P. Rastogi, eds., 83-139 (Wiley-VCH, Weinheim, 2012).

[4] W. Nadeborn, W. Osten, and P. Andrä, "A robust procedure for absolute phase measurement," Opt. Lasers Eng. 24(2-3), 245-260 (1996).

[5] W. Li, T. Bothe, C. von Kopylow, and W. Jütner, "Evaluation methods for gradient measurement techniques," Proc. SPIE 5457, 300-311 (2004).

[6] S. Ettl, J. Kaminski, M. Knauer, and G. Häusler, "Shape reconstruction from gradient data," Appl. Opt. 47(12), 2091-2097 (2008).

[7] H. Zhang, S. Han, S. Liu, S. Li, L. Ji, and X. Zhang, "3D shape reconstruction of large specular surface," Appl. Opt. 51(31), 7616-7625 (2012).

[8] L. Huang, and A. Asundi, "Improvement of least-squares integration method with iterative compensations in fringe reflectometry," Appl. 0pt. 51(31), 7459-7465 (2012).

[9] Y. Tang, X. Su, and S. Hu, "Measurement based on fringe reflection for testing aspheric optical axis precisely and flexibly", Appl. Opt. 50(31), 5944-5948 (2011).

[10] W. Li, and J. Burke, "Highly accurate surface reconstruction for deflectometry," in Proceedings of the 113th Annual Meeting of the DGaO, A23 (DGaO, Eindhoven, 2012).

[11] H. Helmers, A. Boos, F. Jetter, A. Heimsath, M. Wiesenfarth, and A. Bett, "Outdoor Test Setup for Concentrating Photovoltaic and Thermal (CPVT) Systems," Proc. AIP 1407, 175-179 (2011).

[12] P. Su, R. Parks, L. Wang, R. Angel, and J. Burge, "Software configurable optical test system: a computerized reverse Hartmann test," Appl. Opt. 49(23), 4404-4412 (2010).

[13] A. Heimsath, W. Platzer, T. Bothe, and W. Li, "Characterization of Optical Components for Linear Fresnel Collectors by Fringe Reflection Method," in Proceedings of the 14th Solar Paces conference, 1-8 (IEA, Las Vegas, 2008).

[14] www.fringeprocessor.de

[15] A. Heimsath, G. Bern, P. Nitz, "Shape accuracy of solar mirrors comparison of two methods using fringe reflection technique," in Proceedings of the 17th Solar Paces conference, 76 (IEA, Granada, 2011).

[16] M. Sandner, W. Li, T. Bothe, J. Burke, C. von Kopylow, and R. Bergmann, "Absolut-Abstandsreferenz für die Streifenreflexionstechnik zur Verringerung systematischer Messfehler," in Proceedings of the 112th Annual Meeting of the DGaO, A2 (DGaO, Ilmenau, 2011).

[17] J. Burke, K. Wang, and A. Bramble, "Null test of an off-axis parabolic mirror. I. Configuration with spherical reference wave and flat return surface," Opt. Express 17(5), 3196-3210 (2009).

[18] J. Burke, "Null test of an off-axis parabolic mirror. II. Configuration with planar reference wave and spherical return surface," 0 pt. Express 17(5), 3242-3254 (2009). 